\title{
Características del perfil de las personas egresadas del posgrado en Relaciones Internacionales
}

Fonseca-Hernández, Raúl; Cascante-Segura, Carlos Humberto

Características del perfil de las personas egresadas del posgrado en Relaciones Internacionales

Revista Educación, vol. 43, núm. 2, 2019

Universidad de Costa Rica, Costa Rica

Disponible en: http://www.redalyc.org/articulo.oa?id=44058158013

DOI: https://doi.org/10.15517/revedu.v43i2.31870

Esta obra está bajo una Licencia Creative Commons Atribución-NoComercial-SinDerivar 3.0 Internacional. 


\title{
Características del perfil de las personas egresadas del posgrado en Relaciones Internacionales
}

\author{
Characteristics of International Relations Postgraduate Program Graduates
}

Raúl Fonseca-Hernández

Universidad Nacional, Heredia y Universidad de Costa

Rica, Sede Occidente, Costa Rica, Costa Rica

raul.fonseca.hernandez@una.cr

(iD) http://orcid.org/0000-0002-4133-9591

Carlos Humberto Cascante-Segura

Universidad Nacional, Costa Rica

carlos.cascante.segura@una.cr

(iD http://orcid.org/0000-0003-1933-9015

\author{
DOI: https://doi.org/10.15517/revedu.v43i2.31870 \\ Redalyc: http://www.redalyc.org/articulo.oa?id=44058158013
}

(D)

Recepción: 26 Diciembre 2017

Aprobación: 14 Mayo 2019

\section{Resumen:}

El texto tiene por objetivo analizar las características que identifican las personas estudiantes, graduadas, egresadas y las que desertaron del posgrado en Relaciones Internacionales (RI) en Costa Rica. Pretende ser una herramienta para el análisis de la inserción laboral del profesional en RI, su entorno y el contexto en el que actualmente se desenvuelve, para comprender el peso de esta disciplina en el ámbito laboral costarricense. Con tal fin se elaboró una investigación mixta, mediante la aplicación de encuestas a estudiantes y empleadores que permite, luego de aplicar el análisis estadístico, determinar que el posgrado es atractivo para las personas relativamente jóvenes, con carreras base tanto en RI como en otras disciplinas, que trabajan tiempo completo o más, que laboran mayoritariamente en el sector privado. Así mismo, las variables significativas que influyen en graduación de las y los estudiantes son: el género, la formación base, sector donde labore (público o privado), edad, lugar de residencia. Se concluye que la maestría en RI es un elemento que aporta mayor competitividad laboral, sin embargo, las instituciones de educación superior deben hacer un esfuerzo para incorporar en sus planes de estudio el desarrollo de habilidades blandas que el mercado está requiriendo.

Palabras ClaVe: Perfil, Egresado, Posgrado, Relaciones Internacionales.

\begin{abstract}
:
The objective of this study is to identify characteristics of current students, graduates and former students who dropped out of International Relations graduate programs in Costa Rica in terms of future job placement and better understand the weight of this area of study in Costa Rica compared to other disciplines. The results included a combination of surveys completed by both students and employers which, after a statistical analysis, conclude that younger students tend to consider this type of postgraduate program more attractive. Many of these students are employed full time or more and most work within the private sector. Significant variables associated with students that complete and graduate from these programs, such as gender, educational background, labor sector (public or private), age and where a person resides. It is concluded that a master's degree in International Relations is an attribute that makes an individual more marketable. Yet, universities must strive to include soft skills development in the study plans required for today's job market.
\end{abstract}

KEYWORDS: Profile, Graduate, Master's degree program, International Relations.

\section{INTRODUCCIÓN}

Una de las preocupaciones de los últimos años en Costa Rica ha sido la relación entre universidades y mercado laboral. Esta discusión plantea por una parte la capacidad y condiciones del mercado para incorporar graduados universitarios y, por otra, las condiciones que motivan a las personas a continuar estudios de 
posgrado para competir dentro de ese mercado (Salas 2013; Ardila, 2013; Parra, Parra, Vergel, Sánchez, s.f.; Sandoval, Reyes, y Tapia, 2005). Por tal motivo, algunas unidades académicas han iniciado un proceso que se utiliza con mayor frecuencia en el seguimiento de graduados, como instrumento para la mejora continua de la calidad educativa que se brinda en ese nivel de estudios. En esta línea, según Medina et ál. (2005):

Los estudios de seguimiento de egresados son una herramienta importante para analizar los caminos que siguen los nuevos profesionales, no solo en lo que respecta a su inserción laboral, sino al entorno y contexto en que se desenvuelven. Son también mecanismos poderosos de diagnóstico de la realidad con el potencial de inducir en las instituciones la reflexión sobre sus fines y valores, desde que aportan elementos para analizar las ventajas y desventajas de los diferentes modelos y opciones educativas que permitirán su fortalecimiento o revisión (p. 2).

Desde esta perspectiva, el proceso de seguimiento debe ser lo más detallado posible para alcanzar resultados que permitan observar las dificultades de incorporación y desarrollo profesional en el mercado. Esta información permite la generación de distintos perfiles que resultan útiles para mejorar el desarrollo profesional y evitar la deserción de los programas. En general, la línea de técnicas dominante en esta materia ha sido desarrollar estudios sobre la situación de los egresados que han conseguido obtener el título académico. Asimismo, estos se han ordenado por generaciones, de forma que se elaboran encuestas en el primer año tras egresar del programa de estudios (impresiones sobre la educación recibida y situación laboral); en el segundo y quinto año tras la salida del programa para estudiar la capacidad de inserción laboral, con posterioridad al quinto año se efectúan encuestas para determinar la trayectoria laboral del estudiante (Red GRADUA2 y Asociación Columbus, 2006; Gómez, Ortiz y González, 2017).

Desde esta perspectiva, son relevantes los modelos de estudios seguidos en varias universidades latinoamericanas para construir perfiles profesionales basados en estudios más concretos de la realidad laboral en el marco de carreras específicas, como por ejemplo la Universidad de Puebla (Avelino, Cortés, Rosales y Pérez, 2014), Universidad de Talca (Hawes, 2005), Universidad de Ciencias Pedagógicas de Cuba (Barazal y Añorga, 2011), la Universidad de Chile (Zepeda y Herskovic, 2013), así como la Universidad de País Vasco (Araujo, 2003). Estos trabajos tenían en común la necesidad de caracterizar, en la medida de lo posible, al estudiante que egresaba y se graduaba de programas de estudio específicos de dichas universidades con un fin pragmático, dado que a partir de esta información resulta factible determinar aquellas habilidades adquiridas por el estudiante durante su carrera, el entorno laboral a que se iba enfrentar, lo cual permitía establecer rutas de mejora de la educación que debían recibir las próximas generaciones de graduados.

En la literatura sobre la materia se establece que estos estudios requieren la preparación de encuestas que permitan no solo establecer componentes propios del contexto socioeconómico del estudiante, sino también, rescatar elementos concretos sobre la educación que ha recibido dentro del programa de estudios del cual egresa; así como su trayectoria laboral una vez concluido su plan de estudios (Red Gradua2 y Asociación Columbus, 2006; Gómez, Ortiz y González, 2017).

En Costa Rica, la preocupación entre universidades y mercado de trabajo llevó a la conformación del Observatorio Laboral de Profesionales, adscrito al Consejo Nacional de Rectores. Los informes publicados entre 2010 y 2016 dan cuenta de las características y problemáticas de los egresados de carreras de grado (bachillerato y licenciatura) en su proceso de incorporación al mercado laboral, no obstante, por la magnitud de las investigaciones no resulta factible entrar en detalles específicos de cada carrera y mucho menos de planes de estudios particulares (CONARE-OPES, 2018).

En este artículo exponen por primera vez algunas de las conclusiones alcanzadas por la investigación de base ejecutada por Fonseca y Cascante (2015a) titulada Análisis de mercado y factores competitivos de la maestría en Relaciones Internacionales (RI), la cual se efectuó en el marco de los preparativos de reforma de la Maestría en Relaciones Internacionales y Diplomacia de la Universidad Nacional ${ }^{[1]}$. En ella se establecen los perfiles de las personas estudiantes del Posgrado, así como las características sociodemográficas de estos, de igual forma se observa la necesidad de contar con un posgrado el cual integre la virtualidad, que sea más cercano a la provincia de San José, que su maya curricular refleje los grandes problemas de análisis internacional. De tal 
forma, con fundamento en las experiencias y trabajos citados en los párrafos anteriores, el estudio pretendió recabar la información necesaria para plantear rutas para mejorar la gestión académica del posgrado. Sin embargo, existía la dificultad de que en este nunca se había ejecutado este tipo de investigación, de forma que se contaba con una información fragmentaria la cual evitaba establecer los tipos de encuestas a generaciones específicas que se emplean en otros proyectos de este tipo. Adicionalmente, se consideró relevante estudiar las condiciones que producían la éxito y fracaso dentro del programa y no únicamente la situación de aquellas personas que alcanzaron la graduación.

En este contexto, la investigación se planteó a partir de un concepto más amplio de los sujetos a estudiar. Así el objetivo fue establecer las características de las personas que ingresaban a la maestría, conocer los patrones de aquellas que lograban egresar del programa (cumplir todo el plan de estudios), las que lograban graduarse (cumplir plan de estudios y presentar su tesis) y de las que desertaban del programa (no concluían el plan de estudios). Adicionalmente, se propuso indagar sobre los retos que enfrenta un programa de posgrado en la actualidad para ser más competitivo dentro del mercado. El análisis de estas interrogantes permite acceder a una valiosa información para comprender las formas de inserción laboral del profesional en relaciones internacionales, su entorno y el contexto en el que actualmente se desenvuelve.

Para responder estas preguntas, este trabajo se compone de cuatro secciones; en la primera se establece los componentes metodológicos del estudio; el segundo, describe los resultados de la aplicación de un instrumento relativo a las características socioeconómicas de la población estudiantil de la Maestría en Relaciones Internacionales. Dicha sección se divide a su vez en tres apartados que estudian las características de graduados, egresados y desertores del programa. En una tercera sección se presentan los retos académicos que un programa de posgrado en relaciones internacionales debe plantearse como estrategia de desarrollo en el corto, mediano y largo plazo para mejorar su competitividad y la de sus estudiantes. Por último, se presentan las conclusiones de la investigación.

\section{Metodología}

Este estudio constituye una investigación mixta, tipo análisis de caso único, por lo tanto sus conclusiones no pueden generalizarse a otros, sin embargo, puede aportar algunas hipótesis para estudios de mayor amplitud (Díaz, 2011). La investigación mixta, según Guelmes y Nieto (2015) es:

... la búsqueda donde el investigador mezcla o combina métodos cuantitativos y cualitativos y señala como su característica clave el pluralismo metodológico o eclecticismo, lo que resulta en una investigación superior por cuanto utiliza las fortalezas de la investigación cuantitativa y las de la investigación cualitativa combinándolas y minimizando sus debilidades (p. 24).

Para responder a las preguntas se procedió a aplicar dos encuestas separadas, la primera a estudiantes de la Maestría en Relaciones Internacionales de Universidad Nacional de Costa Rica entre 2007 y el 2015 (Fonseca y Cascante, 2015 b); y una segunda a empleadores de los graduados. Estos autores utilizan una consulta a egresados, donde valora aspectos socioeconómicos y otra a empleadores de estas personas para valorar los aspectos que los posgrados en Relaciones Internacionales deberían de ofrecer a sus estudiantes.

La población total de personas inscritas en el posgrado para 2015 era de 287 individuos, cuyos datos de contacto fueron suministrados por el Departamento de Registro; aunque dicha información no contenía distinción entre los graduados, egresados ${ }^{[2]}$ o quienes abandonaron el programa. De forma, que se contaba con una base de datos inicial que abarcaba como variables la cédula, teléfonos y correo de contacto, así como el último año que la Universidad tiene registro de actividad académica en el posgrado.

Finalmente, se aplicó la encuesta a una muestra ajustada de 120 personas. El cálculo de la muestra se efectuó a un $95 \%$ de confianza. La selección de los individuos se elaboró de forma aleatoria. Se utilizó el método de números aleatorios. Se ejecutaron reemplazos aleatorios en los casos en los cuales no se localizaron las personas o cuando se les trataba de contactar sin respuesta en más de tres oportunidades. Sin embargo, la principal 
debilidad de la base de datos inicial fue la desactualización de información de contacto en teléfonos y correos de los egresados, pese a que se comparó la base de datos suministrada por el Departamento de Registro y la Secretaría de la Maestría.

La encuesta fue aplicada vía telefónica entre el once de febrero y el seis de marzo de 2015, y sus resultados almacenados con la ayuda de la plataforma virtual de Google Form. Los horarios de aplicación fueron variados, rotándose entre la mañana, tarde y noche, de lunes a viernes. Únicamente se aplicaron encuestas presenciales a las y los estudiantes del posgrado, que ingresaron en 2014. La información se tabuló y analizó con el apoyo de programas informáticos como Excel 2013 y SPSS 20.

Las variables usadas en el análisis fueron: carreras base, edad, lugar de procedencia, condición laboral, salarios promedio según sector de procedencia, personas que se gradúan, personas que se retiran, diferencias salariales con maestría, salarios por género, habilidades que aporta la maestría y que requiere el mercado laboral, perfil interesado(a), perfil de la persona graduada, perfil del graduado competitivo, perfil del desertor.

La determinación estadística de las variables que influyen en la graduación del estudiantado se desarrolló mediante un modelo econométrico binomial (Jiménez y Salas, 1999) con 96 observaciones, excluyendo las personas que se encuentran actualmente cursando el posgrado y analizando únicamente los casos en los cuales concluyen el plan de estudios, se retiran y egresan. Se utilizó el programa Eviews 9 para el cálculo del modelo. Se analiza la covarianza de las variables y la probabilidad de proyección de las estas a un $95 \%$ de confianza. Por otra parte, al analizar el fenómeno de la deserción del posgrado se recurre a estadística descriptiva contextualizada a partir de la serie de preguntas de índole cualitativa que se incluyeron dentro de la encuesta descrita.

La encuesta de empleadores fue aplicada a una población de 47 patronos entre los meses de abril y mayo de 2015, principalmente vía telefónica y por correo electrónico, con base a los datos aportados por los y las personas entrevistadas para la realización del estudio de mercado y otras instituciones de las cuales se sabe que contratan personal en áreas afines a RI (Fonseca y Cascante, 2015b).

Por último, para la determinación del perfil de la persona graduada, se calcula el coeficiente de correlación de Person, el cual mide la relación entre dos variables. La correlación se ubica entre un rango de - 1 y 1 , un valor de 1, implicaría una correlación perfecta del 100\% entre las variables en análisis (Fernández y Díaz, 2001).

\section{Resultados}

\section{Características generales de las entrevistadas}

La maestría en Relaciones Internacionales es un programa abierto a la interdisciplinariedad, de tal forma a lo largo del periodo bajo estudio ha captado igual proporción de estudiantes con formación base en relaciones internacionales y de otras carreras. Así, al 50\% de estudiantes provenientes de las relaciones internacionales, se adicionan los que provienen de periodismo (9\%), derecho (8\%) y administración (7\%). En menor medida, ingresan estudiantes con formación base en educación, política, comercio exterior, relaciones públicas, medicina, inglés, turismo, historia, contaduría pública, sociología, psicología, arquitectura, enfermera, geofísica, filosofía, economía, antropología, ingeniería agrícola e ingeniería forestal (ver Gráfico 1). Los datos demuestran que el posgrado en RI atrae a estudiantes de diversas disciplinas de las ciencias sociales y humanidades, no obstante, tampoco excluye la participación de estudiantes de carreras vinculadas con ciencias aplicadas como ingeniería y medicina.

Por otra parte, aunque atrae estudiantes de diferentes grupos etarios, resulta especialmente atractivo para personas cuyas edades rondan entre los 22 y los 30 años. Este aspecto resulta relevante, pues supone que el estudiantado tiene facilidad para desenvolverse en ambientes virtuales y tiende a ser exigente con la calidad del 
servicio que adquiere. Asimismo, el posgrado es elegido predominantemente por mujeres (66\%) que hombres (34\%) (Gráfico 1).

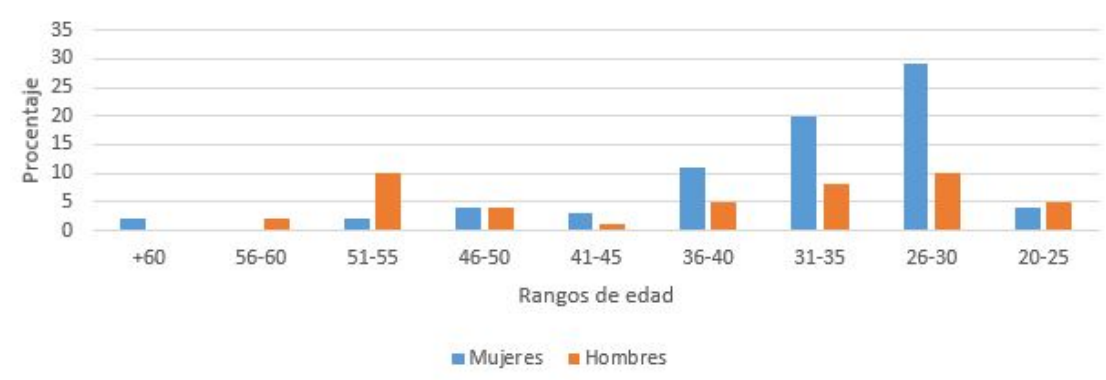

\section{GRÁFICO 1}

Edades promedio según género

Fuente: Elaboración propia a partir de archivo de la Maestría en Relaciones Internacional, UNA.

El alumnado que habita en la Gran Área Metropolitana (GAM) reside mayoritariamente en los cantones de Heredia y San José (Ver Tabla 1).

TABLA 1

Lugar de residencia del estudiantado de la muestra divididos según cercanía con la ciudad de Heredia

\begin{tabular}{|c|c|c|c|c|c|}
\hline \multicolumn{2}{|l|}{ Grupo 1} & \multicolumn{2}{|l|}{ Grupo 2} & \multicolumn{2}{|l|}{ Grupo 3} \\
\hline Cantón & $\begin{array}{l}\text { Total de } \\
\text { estudiantes }\end{array}$ & Cantón & $\begin{array}{l}\text { Total de } \\
\text { estudiantes }\end{array}$ & Cantón & $\begin{array}{l}\text { Total de } \\
\text { estudiantes }\end{array}$ \\
\hline Heredia & 30 & San José & 27 & Nicoya & 1 \\
\hline Tibás & 5 & Cartago & 7 & $\begin{array}{l}\text { Pérez } \\
\text { Zeledón }\end{array}$ & 1 \\
\hline Belén & 4 & Alajuela & 6 & Poás & 1 \\
\hline $\begin{array}{l}\text { San } \\
\text { Pablo }\end{array}$ & 2 & Moravia & 6 & Puriscal & 1 \\
\hline $\begin{array}{l}\text { Santo } \\
\text { Domingo }\end{array}$ & 2 & Desamparados & 4 & $\begin{array}{l}\text { San } \\
\text { Ramón }\end{array}$ & 1 \\
\hline Barva & 1 & Montes de Oca & 4 & $\begin{array}{l}\text { Valverde } \\
\text { Vega }\end{array}$ & 1 \\
\hline $\begin{array}{l}\text { San } \\
\text { Isidro }\end{array}$ & 1 & Grecia & 3 & Acosta & 1 \\
\hline & & La Unión & 3 & El Guarco & 1 \\
\hline & & Santa Ana & 2 & & \\
\hline & & Coronado & 2 & & \\
\hline & & Curridabat & 1 & & \\
\hline & & Escazú & 1 & & \\
\hline & & Zapote & 1 & & \\
\hline $\begin{array}{l}\text { Total } \\
\text { (abs. /rel.) }\end{array}$ & $45 / 37,5$ & $\begin{array}{l}\text { Total } \\
\text { (abs./rel.) }\end{array}$ & $67 / 55,8$ & $\begin{array}{l}\text { Total } \\
\text { (abs./rel.) }\end{array}$ & $8 / 6,7 \%$ \\
\hline
\end{tabular}

Fuente: Elaboración propia

La mayoría de las personas entrevistadas están laborando y lo hacen a tiempo completo. De los entrevistados, el $91 \%$ tiene un trabajo remunerado (109 personas) y solo el $9 \%$ no lo hace actualmente por situaciones personales o permisos de trabajo especiales. Del total de personas que están empleadas, el 83\% labora a tiempo completo, o incluso, un $9 \%$ trabaja más del tiempo completo. El restante $8 \%$ labora menos de tiempo completo o está jubilado.

Las personas entrevistadas desempeñan labores de análisis y gerencial. La mayoría ejecuta labores analíticas, de apoyo, técnicas y de investigación. En menor proporción desarrollan labores gerenciales. El posgrado 
debe cumplir con las expectativas de todos los interesados, por tanto debe dotar de herramientas técnicas y prácticas para dar soporte a labores de analíticas, así como, brindar conocimientos y habilidades pertinentes a puestos gerenciales, de dirección de personal y gestión estratégica.

Los salarios promedio son más competitivos en el sector público, a pesar de que en este sector trabaja una menor cantidad relativa de personas en relación con el total de entrevistados. El 35\% labora para instituciones gubernamentales, cerca del 38\% lo hace para empresas privadas, mientras que el 6\% está vinculado a organizaciones sin fines de lucro y únicamente 4 personas (3\%) indican tener una empresa propia tipo consultora. El promedio de los salarios de los entrevistados ronda los $\$ 1.850$ (USD) por mes, la mayoría (40\%) gana entre \$ 924 y $\$ 1.850$ mensuales, el 30\% de los entrevistados tienen salarios superiores que van desde $\$ 1.850$ y hasta los $\$ 8.323$ por mes. En el sector público los salarios promedio rondan $\$ 2.400$, posiblemente la base salarial se incrementa con pluses como dedicación exclusiva, anualidades, entre otros. En contraposición, en el sector privado el sueldo medio está cercano al \$1.850 por mes. El Gráfico 2 muestra los salarios promedio generales y los que se registran por sector de las y los estudiantes del posgrado.

(a) Distribución general
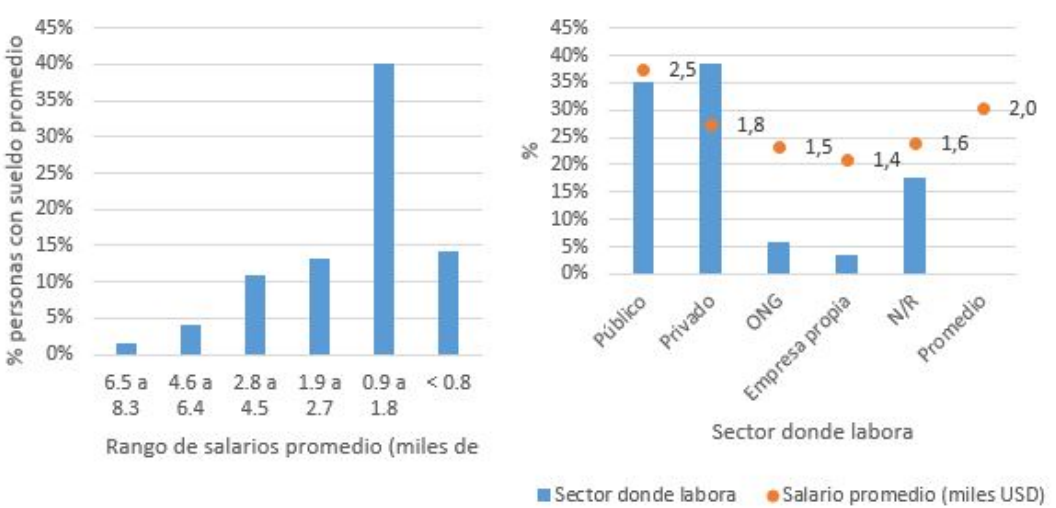

GRÁFICO 2

Distribución general y por sector de los salarios promedios de las personas matriculadas en la maestría de RI (2007-2015) Fuente: Elaboración propia.

\section{DESEMPEÑo DE LOS ENTREVISTADOS EN EL POSGRADO}

En la presente sección se analizan las características particulares de los entrevistados según su situación académica en la maestría. Cabe destacar que se entrevistó a personas matriculadas que concluyeron el programa académico y obtuvieron su título respectivo; las que egresaron, es decir, que completaron los cursos pero no efectuaron su trabajo final de graduación y las que desertaron de la maestría.

En el período en análisis es posible establecer tres perfiles, los cuales coinciden con lo propuesto por Rodríguez y Valenzuela (2017). Un primer perfil corresponde al estudiantado que logra concluir todo el posgrado (30\%), uno asociado a personas que ingresan al posgrado y concluye el plan de estudios, pero no logra graduarse pues no presentan tesis (32\%) y las personas que no concluyen el plan de estudios y deciden abandonar el programa (38\%). Es decir, de un grupo de 20 personas que inician la maestría, únicamente se graduarán seis personas y catorce desertarán o no lograrán concluir su trabajo final de graduación. Esto significa un serio problema para la sostenibilidad financiera y continuidad del posgrado, considerando el número mínimo de estudiantes reglamentario que se requiere para abrir un curso. 
A continuación, se desarrolla cada perfil según los datos de la encuesta aplicada, siguiendo a Fonseca, Cascante, Arce y Abarca (2016).

\section{1) Características del perfil de la persona graduada}

En el caso de las personas graduadas, se calculó el coeficiente de correlación de Person, el cual permite determinar la significancia y el peso estadístico de cinco factores: el género, la formación base, el sector laboral (público o privado), la edad y el lugar de residencia. El modelo compara los cinco factores y asigna un peso estadístico a cada uno de ellos, excluyendo de la muestra para este ejercicio aquellos casos que se encuentran actualmente con el programa en curso (ver Tabla 2).

TABLA 2

Valores del coeficiente de correlación de Person entre las variables que caracterizan a la persona graduación de las y los estudiantes del posgrado.

\begin{tabular}{|l|l|l|l|l|}
\hline Género & $\begin{array}{l}\text { Formación } \\
\text { base (al } \\
\text { menos } \\
\text { bachillerato) }\end{array}$ & $\begin{array}{l}\text { Sector } \\
\text { (público- } \\
\text { privado) }\end{array}$ & Edad & $\begin{array}{l}\text { Lugar de } \\
\text { residencia }\end{array}$ \\
\hline $58,85 \%$ & $35,83 \%$ & $7,38 \%$ & $3,57 \%$ & $1,26 \%$ \\
\hline
\end{tabular}

Fuente: Elaboración propia.

En este sentido, existe una mayor probabilidad de que las mujeres logren graduarse del posgrado, en esta línea, de las 61 mujeres encuestadas 21 obtuvo el título (34,4\%); mientras que 8 de los 35 hombres encuestados alcanzó dicho objetivo (22,9\%).

La formación base en relaciones internacionales constituye un condicionante importante para aquellos casos de éxito en el programa, pues aumenta en un 35,83\% la probabilidad de graduación de una persona estudiante de la maestría, de tal forma, que quienes provienen de otras carreras, tienden con mayor frecuencia a no concluir sus estudios.

El sector laboral (público o privado) de donde proviene el estudiantado también explica una mayor probabilidad de conclusión de la maestría. En este sentido, existe una mayor tendencia a concluir el plan de estudios si se proviene del sector público, dado posiblemente a que existen incentivos salariales asociados a la obtención del título. De tal forma el 58,6\% de los graduados trabajaba en el sector público, esta condición resultaba más determinantes para los hombres, pues el 75 de los graduados de ese género trabajaba en ese sector, lo que contrastaba con apenas el 52,3\% de las mujeres. Asimismo, el 45,9\% de estudiantes que trabajaba en el sector público logró graduarse, lo que superaba por mucho el 17,6\% de aquellas y los estudiantes que trabajaban en el sector privado.

Por otra parte, el grupo etario o lugar de residencia del estudiante influye relativamente poco en la graduación de las personas, de tal forma que independientemente de la edad que se tenga, la probabilidad de conclusión de la maestría tiende a ser la misma. El lugar de residencia es una variable que tiene relativamente poca influencia en la graduación de los encuestados, de la clasificación hecha anteriormente (Tabla 1), de tal forma el 25,8 de residentes en los cantones del grupo 1, el 31,6 del grupo 2 y el 28,6\% del grupo 3 lograron graduarse. En este sentido, tienen relativamente la misma probabilidad de graduación aquellas personas que habitan cerca de la ciudad de Heredia o fuera de ella. En este punto debe advertirse que el modelo se enfoque en observar las variables significativas y el grado de asociación entre estas y la probabilidad de graduación, excluyendo de su análisis el razonamiento que explicaría la deserción de las y los estudiantes de la maestría. 
El perfil desarrollado para estos casos permite apreciar como en posgrado abierto a una diversidad de carreras, las y los estudiantes provenientes de las relaciones internacionales tienen mayores posibilidades de éxito. En esta línea, la toma de decisiones tiene que orientarse a equiparar las destrezas y conocimiento técnico que las y los estudiantes que no provienen de esta disciplina tengan mejores posibilidades de concluir y graduarse. Con las variables estudiadas no resulta posible determinar cuáles son las causas para que las mujeres tengan mejores porcentajes de graduación que los hombres; pero si como el permanecer como funcionario público aumenta las posibilidades de éxito dentro del programa.

\section{2) Características de las personas que concluyen el plan de estudios, pero no se gradúan}

Para tratar esta población se recurrirá exclusivamente a la estadística descriptiva con cruce de variables, dado que la cantidad de observaciones es limitada para poder plantear un modelo binomial. 31 personas lograron concluir su plan de estudios, pero aún no completan el respectivo trabajo final de graduación, 61,2\% de mujeres y 38,8 hombres; un 51,6\% provienen de la carrera de relaciones internacionales, porcentaje menor al de graduados. Al cruzar la variable género con la formación base, puede notarse como $52,6 \%$ de las mujeres en esta categoría provienen de relaciones internacionales; muy similar al porcentaje de hombres que alcanza el 50\%. La conclusión del plan de estudios se encuentra, a diferencia de la graduación, se encuentra, por ende, menos influenciado por la carrera base de las y los estudiantes, aunque se mantiene como un factor relevante.

Por otro lado, 58,1\% de esta población se encontraba trabajando en el sector privado, apenas un 32,3\% en el sector público y un 9,6\% no respondieron la pregunta. En esta línea, la pertenencia al sector público se constituye en un elemento relevante para explicar, por lo menos, desde el punto de vista de incentivos, porque este grupo no desarrolla su trabajo final de investigación y logra graduarse.

Asimismo, otro factor que comienza a influir en la conclusión del posgrado, es la cercanía de su residencia con la sede donde se imparte el posgrado. En este sentido, siguiendo la clasificación establecida en la Tabla 1, un $41,9 \%$ del grupo 1, un 31\% del grupo 2, lograron concluir los cursos del plan de estudios. Ningún estudiante del grupo 3 alcanzó ese resultado. De tal forma, las y los estudiantes de grupo 1, es decir las y los estudiantes con residencia más cercana a la sede del posgrado tienen más éxito en la conclusión de su plan de estudios que los incluidos dentro de los otros grupos.

En este sentido, el perfil de esta población obliga a que se desarrollen mecanismos dirigidos a apoyar los procesos de investigación de las y los estudiantes, pues sus problemas -de acuerdo con la evidencia-. En este sentido, de las 24 personas que habían concluido el plan de estudios y respondieron a la pregunta: ¿por qué no concluyó el programa de maestría?, la mitad hace referencia a la necesidad de contar con más guía académica en el proceso y la otra mitad a razones personales. Desde esta perspectiva, resulta indispensable para los posgrados diseñar un acompañamiento que permite incorporar a las y los estudiantes a procesos de investigación en marcha.

\section{3) Características de las personas que abandonan el plan de estudios}

Al igual que en el perfil anterior se emplea únicamente el cruce de variables con estadística descriptiva. El perfil de esta población de la muestra que no concluyó el plan de estudios se encuentra compuesto por un $58,8 \%$ de mujeres y 41,2 de hombres. En términos generales, el 30\% provienen de una formación en relaciones internacionales, lo cual contrasta con los perfiles anteriores, donde la población que consigue avanzar más en el programa e incluso graduarse proviene mayoritariamente de esta carrera. En esta línea, el conocimiento base, el agrado por las temáticas abordadas en los diversos cursos y los conocimientos metodológicos aplicados a la disciplina constituyen componentes relevantes para la permanencia en el programa. 
Por otra parte, el 55,6\% de las personas que conforman este grupo trabajaban en el sector privado, $27,8 \%$ laboraban en el sector público, mientras que $16,6 \%$ no respondió a la pregunta o se encontraba sin trabajo. En esta línea, los incentivos monetarios y de crecimiento laboral que brinda el sector público son un factor relevante para que las y los estudiantes avancen en el programa. Al no ser algo controlable por el programa, esto obliga a desarrollar mecanismos que hagan atractivo a las y los estudiantes que laboran en el sector privado para que permanezcan el programa, lo cual se abordará en el último acápite de este artículo.

Otro elemento relevante es la distancia con respecto a la sede en que se brinda el posgrado. De las y los estudiantes estratificados por cercanía, aquellos que pertenecen al grupo 1 son los que menos se retiran antes de concluir el plan de estudios con un 32,3\%; mientras que 36,2\% del grupo 2 y el 71,4\% del grupo tres desertan del programa. Junto con la distancia, la edad constituye un elemento diferenciador, pues una considerable cantidad tiene edades superiores a los 30 años, así que a mayor edad menos posibilidad de concluir el programa.

Asimismo, al analizar las respuestas cualitativas a la pregunta ¿por qué no concluyó el programa de maestría? las respuestas se concentran en la existencia de factores externos al programa, así como otras vinculados a la necesidad de este se adapte a requerimiento de la población que no tiene como base la disciplina de las relaciones internacionales, además, se plantea que debe brindar ventajas para competir y crecer en el mercado laboral.

\section{LOS RETOS DE UN PROGRAMA DE POSGRADO EN RELACIONES INTERNACIONALES: COMPETITIVIDAD EN EL MERCADO Y CALIDAD ACADÉMICA}

Los resultados de la encuesta esbozados a lo largo de este trabajo señalan la necesidad de responder cuáles son los componentes que un programa de posgrado en relaciones internacionales puede brindar para que un profesional sea más competitivo dentro del mercado. De acuerdo con el instrumento que se aplicó a empleadores, el título de maestría es un elemento diferenciador, sin importar la formación base o el género, permite acceder a un mayor ingreso y mejores oportunidades labores. En consecuencia, la obtención del título de maestría tiene tendencia observable mayor con aumentos en la compensación salarial y asensos laborales sin importar el énfasis que curse o el sector donde labore, como lo demuestra la encuesta aplicada estudiantes graduados de la maestría en relaciones internacionales en sus diferentes énfasis ${ }^{[3]}$. Las personas que obtienen el certificado ganan más en comparación con aquellas que no concluyen sus estudios, incluso, tienen mayor ingreso que aquellas personas que egresan de la maestría. La diferencia es significativa tanto en el sector público como privado (series 5 y 6 respectivamente). De igual forma, la tendencia se mantiene con independencia de los énfasis que curse (ver Gráfico 3). 


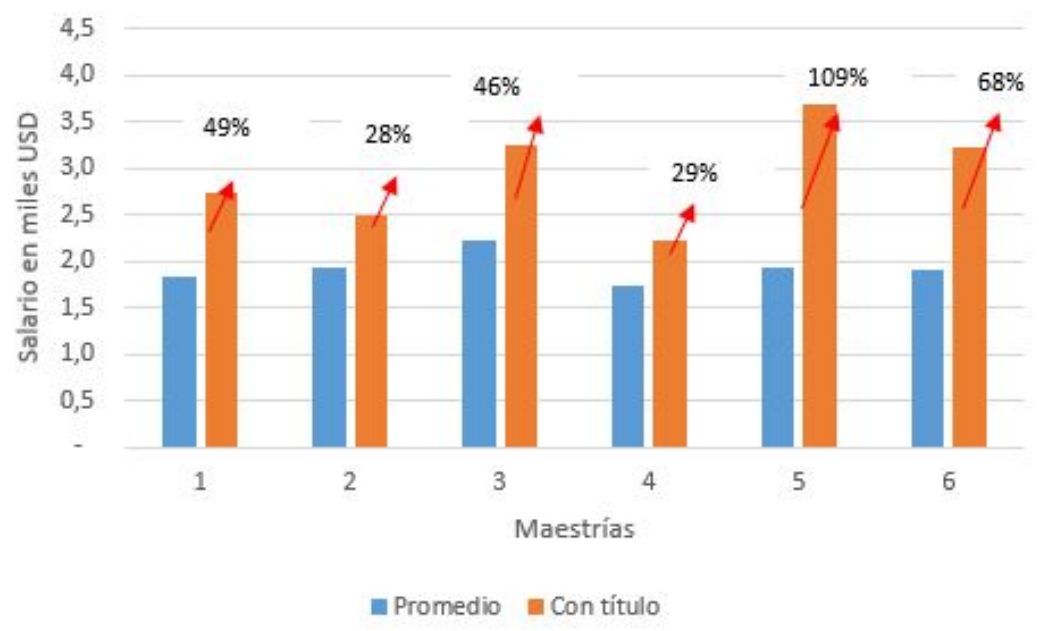

\section{GRÁFICO 3}

Salario promedio de graduados(as) y no graduados(as) según el énfasis y sector para el cual laboran, 2007-2015

Fuente: Elaboración propia.

Resulta notorio que los profesionales que se gradúan de la maestría con formación base en relaciones internacionales son quienes registran salarios más competitivos. Para estos entrevistados concluir su maestría les significó un 49\% de salario adicional, pasan de ganar en promedio 1.800 USD sin el posgrado, a 2.700 USD una vez finalizada la maestría (serie uno). Las personas graduadas del posgrado con formación base en relaciones internacionales tienen un salario en promedio $28 \%$ mayor al de otros profesionales que obtienen el diploma. Los graduados de la maestría en relaciones internacionales con otra formación base, también ven incrementados sus ingresos, pero en menor proporción (ver Gráfico 4).

Sin embargo, las personas de otras carreras que no concluyen el posgrado tienen mejor salario que profesionales en relaciones internacionales en igual condición. Las personas con otra formación base obtienen, en promedio, 54\% más que los internacionalistas que no concluyen el posgrado. El Gráfico 4 muestra las diferencias salariales promedio entre los graduados y no graduados que tienen como formación base las relaciones internacionales y otras especialidades. 


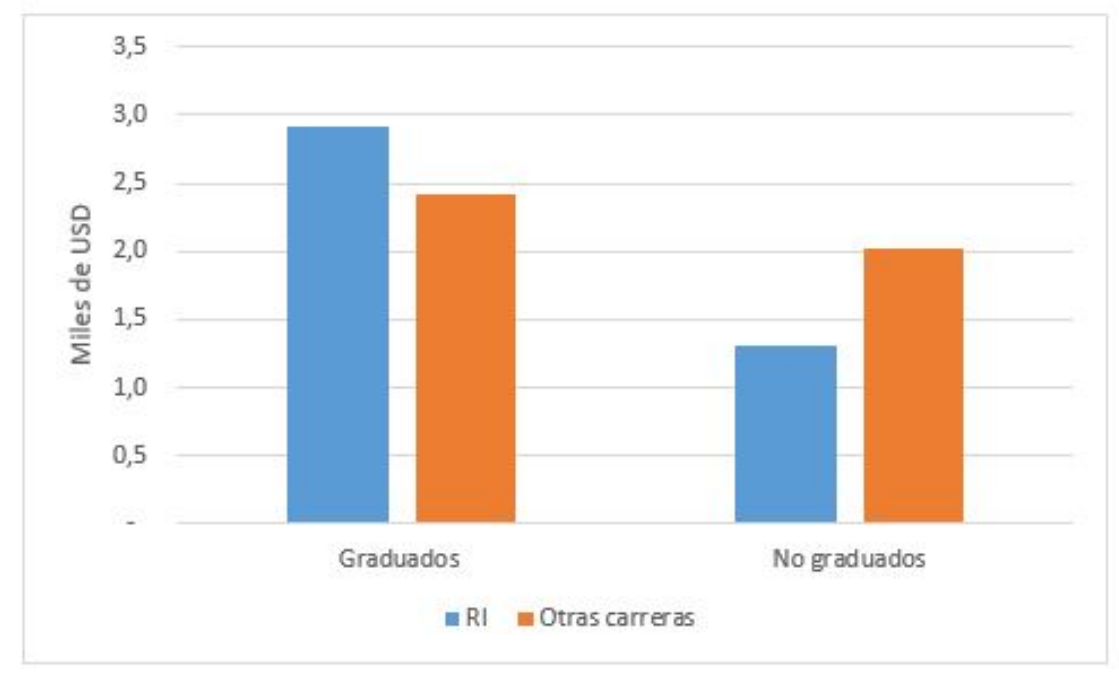

GRÁFICO 4

Salario promedio de graduados(as) y no graduados(as) según su área de formación base. 2007-2015. Fuente: Elaboración propia.

El posgrado garantiza mejor remuneración en ambos géneros, sin embargo, persiste una importante brecha de género. Las mujeres ganan en promedio menos que los hombres con igual título. El Gráfico 5 muestra esta tendencia.

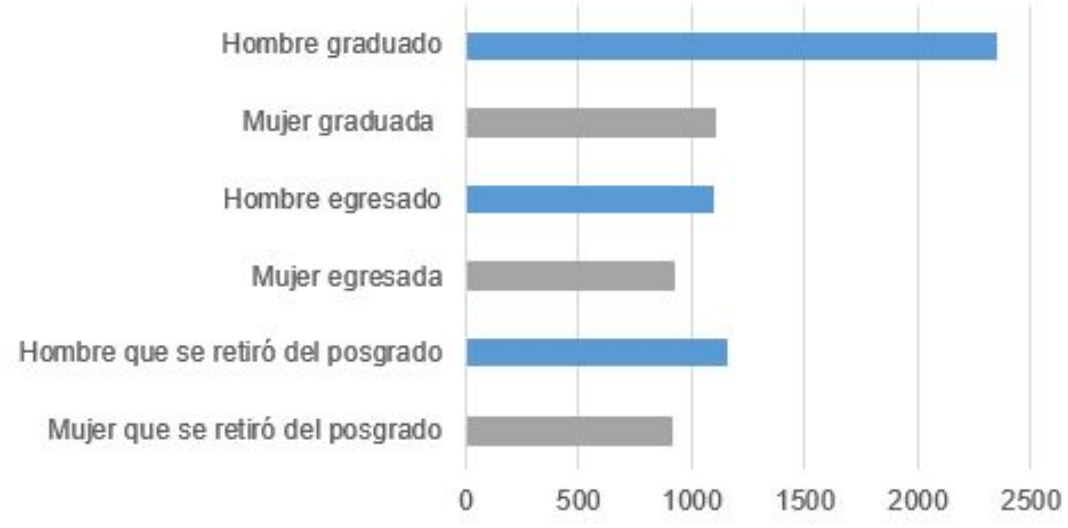

GRÁFICO 5

Salario promedio según el género. 2007-2015

Fuente: Elaboración propia.

Resulta relevante señalar que los empleadores encuestados coincidieron, en general, que las características del profesional competitivo y que, por ende, es mejor valorado por el mercado están asociadas principalmente a las competencias y habilidades blandas. En otros términos, la diferenciación que aporta el título de posgrado en relaciones internacionales está condicionada a que este forme al estudiante con sólidas bases conceptuales y bajo métodos educativos enfocados en las herramientas prácticas-aplicadas.

De forma transversal, el programa debe desarrollar habilidades blandas en el manejo de personal, mejorar la comunicación oral y escrita, así como trabajar en equipo apoya en las calificaciones profesionales. Existen, además, elementos que no necesariamente pueden ser entregados por el posgrado como el dominio de un segundo idioma y el manejo de uno o dos softwares diferentes al Office. El Gráfico 6 muestra los 
conocimientos que fueron valorados por los empleadores como componentes que afectan positivamente la condición salarial. El tener conocimiento de un segundo idioma y poder ejecutar múltiples tareas al mismo tiempo, es mejor valorado en el mercado laboral que la obtención de maestría. Una combinación de ambas explica a un trabajador altamente calificado.

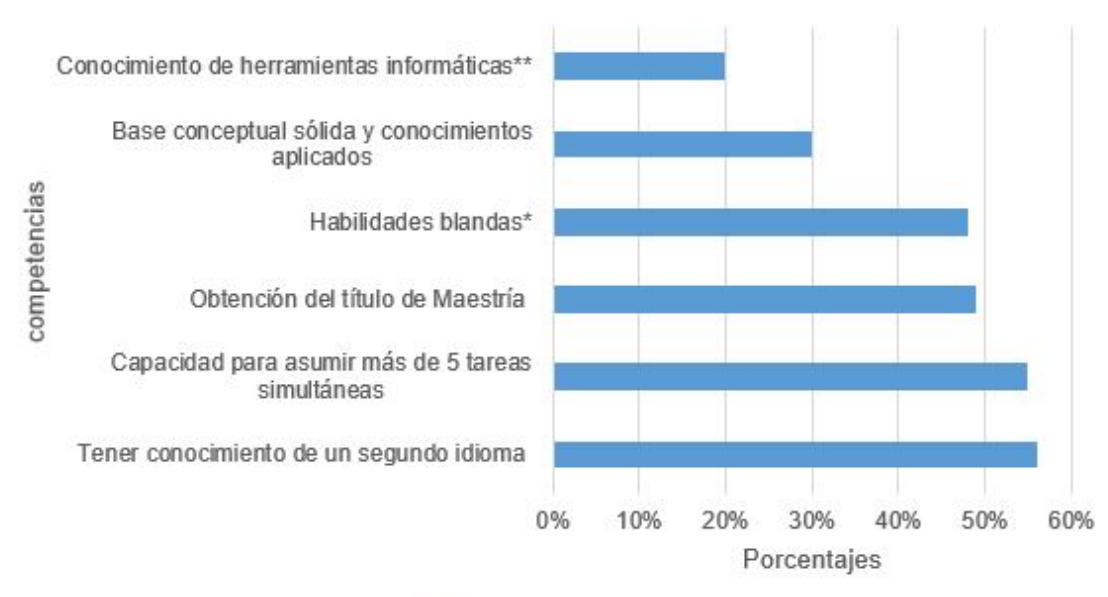

- Factores competitivos

GRÁFICO 6

Competencias que aportan una mayor competitividad al profesional en términos salariales. Nota:

* Habilidades como dirección de personal, técnicas de comunicación oral y escrita, así como trabajo en equipo. ${ }^{* *}$ Conocimientos en herramientas informáticas diferentes de Office. Fuente: Elaboración propia.

En esta línea, los datos y las expresiones de las personas empleadoras coinciden con motivos expresados por el estudiantado que dejó el programa previo a la conclusión del plan de estudios, al señalar la necesidad de encontrar algunos programas que les volvían más competitivos en el mercado. De tal forma, el reto de una maestría en relaciones internacionales consiste en, sin dejar el núcleo duro de la disciplina, aportar componentes de habilidades blandas ligadas a esta, tales como la capacidad de análisis estratégico, la resolución de conflictos y la calidad de redacción de diversos documentos. Además, resulta fundamental la introducción de herramientas informáticas en los cursos que así lo permitan, dado que esto ampliará las posibilidades del alumnado para ser competitivos en el mercado laboral.

\section{CONSIDERACIONES FINALES}

Contar con el perfil del estudiantado permite tener una base actualizada para apoyar procesos de reforma y manejo de la malla curricular y el contar con facilidades educativas que apoyen al estudiante, lo cual facilita que estas personas permanezcan y concluyan sus estudios de posgrado. No obstante, estos estudios se encuentran limitados en el caso costarricense por los problemas de seguimiento de egresados que puedan tener las universidades públicas. Lo anterior limita el acceso a información actualizada de las personas

En el caso concreto de la Maestría en Relaciones Internacionales debe señalarse que su particularidad consiste en el carácter multidisciplinario de sus estudiantes, el cual el propio programa fomenta al no establecer límites respecto de la base académica que debe tener el estudiante que se postula al ingreso. Sin embargo, debe desarrollar las condiciones para que estudiantes ajenos a la disciplina, pues como quedo evidenciado en este estudia existe una tendencia a que este grupo no tenga éxito dentro del programa (obtención del título académico). Desde esta perspectiva, resulta indispensable un diseño de cursos para 
fomentar en el estudiantado que no proviene de relaciones internacionales los conocimientos teóricos y metodológicos mínimos para que aborde con mayor facilidad la disciplina.

Por otra parte, el desplazamiento de estudiantes a la sede del posgrado constituye un obstáculo para su permanencia. De tal forma, deben desarrollarse estrategias que reduzcan el impacto de dicha situación, por ejemplo, herramientas para la virtualización y la planificación de horarios concentrados de clases, para reducir la cantidad de desplazamientos que debe desarrollar el alumnado a lo largo de la duración del plan de estudios. Asimismo, la virtualización puede ser un elemento adicional que contribuya a la internacionalización del posgrado (Aguilar y Riveros, 2017).

El análisis elaborado pone de manifiesto que el incentivo económico resulta fundamental para que las y los estudiantes completen el posgrado, un buen indicador se encuentra constituido por el mejor porcentaje de graduación que tienen las personas que laboraban en ese momento en instituciones públicas; muy diferentes a la situación de los que trabajaban en el sector privado. Desde esta perspectiva, para reducir la propensión de estos último a dejar sus estudios el posgrado debe desarrollar una serie de elementos de competitividad valiosos en el sector privado, tales como brindar al estudiante una serie de habilidades blandas transversales al posgrado, así como un mayor acceso a herramientas informáticas puede compensar en parte las diferencias observadas en este trabajo.

\section{ReFERENCiAS}

Aguilar, Y.y Riveros, A. S. (2017). La internacionalización de la educación superior: Concepto y evolución del modelo en la Universidad de Costa Rica. Revista Educación, 41(1), 1-31.

Araujo, A, Ibáñez, P., Landeta, J.y Ranguelov, S. (2003). Análisis de la flexibilidad de la oferta de formación en gestión del país Vasco. Cuadernos de gestión, 3(1-2), 101-119. Recuperado de https://dialnet.unirioja.es/servlet/articu lo? codigo $=859373$.

Ardila, L. (2013). Caracterización del mercado laboral de los recién egresados de la educación superior: el caso de la Pontificia Universidad Javeriana. Bogotá: Editorial Pontificia Universidad Javeriana.

Avelino, J., Cortés, J., Rosales, S. y Pérez, D. (2014). Articulando el perfil del egresado: competencias adquiridas, en la licenciatura de medicina, UAP. México: Universidad Autónoma de Puebla.

Barazal, A., y Añorga, J. (2011). El perfil del egresado de la Maestría en enfermería y su vinculación con los principios de la Educación Avanzada. Revista Cubana de Enfermería, 27(2), 115-121.

CONARE-OPES. (2018). Seguimiento de la condición laboral de las personas graduadas 2011-2013 de las universidades costarricenses. San José, Costa Rica: CONARE.

Díaz, S., Mendoza, V. y Porras, C. (2011). Una guía para la elaboración de estudios de caso. Revista Razón y Palabra, 75 (1), s.n. Recuperado de http://www.razonypalabra.org.mx/N/N75/varia_75/01_Diaz_V75.pdf

Fernández, P y Díaz, P. (2001). Relación entre variables cuantitativas. Unidad de Epidemiología Clínica y Bioestadística, (4), 141-144. Recuperado de https://www.fisterra.com/mbe/investiga/var_cuantitativas/var_cuantitativas2.p df

Fonseca, R., Cascante, C., Arce, Y. y Abarca, L. (2016). Characterization of Postgraduate Programs Offers in International Relations in Costa Rica and Internationally. Revista Electrónica Educare, 20(3), 1-22. doi: http:/ /dx.doi.org/10.15359/ree.20-3.3

Fonseca, R., y Cascante, C (2015a). Análisis de mercado y factores competitivos de la maestría en Relaciones Internacionales (RI). Documento de trabajo. Costa Rica: Universidad Nacional.

Fonseca, R y Cascante, C. (2015 b). Valoración del mercado laboral porparte de las personas empleadoras de profesionales en el área de RI. Documento de trabajo. Costa Rica: Universidad Nacional.

Guelmes, E. y Nieto, L. (2015) Algunas reflexiones sobre el enfoque mixto de la investigación pedagógica en el contexto cubano. Revista Cientifica de la Universidad de Cienfuego, 7(2), 23-29. 
Gómez, H., Ortiz, E. y González, M. (2017). El estudio de egresados para la mejora continua de las Universidades: Estudio de caso de la Ingeniería en Computación. Revista Iberoamericana para la Investigación y el Desarrollo Educativo, 14(7), 1-23 Recuperado de http://www.scielo.org.mx/pdf/ride/v7n14/2007-7467-ride-7-14-00162 .pdf.

Hawes, G. (2005). Construcción de un perfil profesional. Talca: Universidad la Talca, Instituto de Investigación y Desarrollo Educacional.

Jiménez, J., y Salas, M. (1999). Análisis económico de la elección de carrera universitaria. Un modelo "logit" binomial de demanda privada de educación. España: Instituto Valenciano de Investigación.

Medina, M., Mosconi, E., Albarracín, S., Papel, G., Coscarelli, N., Rueda, L. y Irigoyen, S. (2005). Modelo para evaluación y seguimiento de los egresados aplicado en la facultad de odontología de la UNLP. Argentina: Universidad Nacional de la Plata.

Parra, H. M., Vergel, M. y Sánchez, J. V. (s. f.). Hacia un modelo para evaluar la pertinencia social en la oferta académica de la Universidad Francisco de Paula Santander. Colombia: Universidad Francisco de Paula Santander.

Red GRADUA2 y Asociación Columbus (2006). Manual de instrumentos y recomendaciones sobre el seguimiento de egresados. México: Instituto Tecnológico de Monterrey.

Rodríguez, K. L. y Valenzuela, M. G (2017). Estudio de seguimiento a personas graduadas de postgrados de la Facultad de Ciencias Sociales y Humanísticas (FCSH) de la Escuela Superior Politécnica del Litoral (ESPOL). Revista Educación, 41(1), 1-18

Salas, I. (2013). Los profesionistas universitarios y el mercado laboral mexicano: convergencias y asimetrías. Revista de Educación Superior, 165, 63-81.

Sandoval, R. J., Reyes, J. M. y Tapia, J. L. (2005). Análisis de la oferta educativa a nivel de maestría en México. UPIICSA, 13(38), 2-8.

Zepeda, G., y Herskovic, P. (2013). Perfil de características ideales del docente de postgrado de pediatría. Revista chilena de pediatria, 84(2), 152-159.

\section{Notas}

[1] La Maestría en Relaciones Internacionales es un programa adscrito a la Escuela de Relaciones Internacional de la Universidad Nacional. Este fue creado a finales de la década de 1980 y se encuentra abierto a profesionales de Ciencias Sociales y Humanidades; así como profesionales de otras ramas del conocimiento.

[2] Personas que completaron el plan de estudio pero no concluyeron el trabajo final de graduación o la tesis respectiva.

[3] Por razones de confidencialidad de la información no se pueden indicar los énfasis de las diferentes maestrías, estas se mencionan con los números 2,3 y 4 .

\section{BY-NC-ND}

